TITLE:

\title{
Simulating Adaptive Human Bipedal Locomotion Based on Phase Resetting Using Foot- Contact Information
}

\section{$\operatorname{AUTHOR}(\mathrm{S})$ :}

Aoi, Shinya; Ogihara, Naomichi; Sugimoto, Yasuhiro; Tsuchiya, Kazuo

\section{CITATION:}

Aoi, Shinya ... [et al]. Simulating Adaptive Human Bipedal Locomotion Based on Phase Resetting Using Foot-Contact Information. Advanced Robotics 2008, 22(15): 1697-1713

\section{ISSUE DATE:}

2008

URL:

http://hdl.handle.net/2433/84829

\section{RIGHT:}

(C) 2008 Brill Academic Publishers; This is not the published version.

Please cite only the published version.; この論文は出版社版でありませ ん。引用の際には出版社版をご確認ご利用ください。 


\title{
Simulating adaptive human bipedal locomotion based on phase resetting using foot-contact information
}

\author{
Shinya Aoi ${ }^{1}$, Naomichi Ogihara ${ }^{2}$, Yasuhiro Sugimoto ${ }^{1}$, and Kazuo Tsuchiya ${ }^{1}$ \\ ${ }^{1}$ Dept. of Aeronautics and Astronautics, Graduate School of Engineering, Kyoto University, \\ Yoshida-honmachi, Sakyo-ku, Kyoto 606-8501, Japan \\ 2 Dept. of Zoology, Graduate School of Science, Kyoto University, Oiwake-cho, Kitashirakawa, Sakyo-ku, \\ Kyoto 606-8502, Japan \\ Email: shinya_aoi@kuaero.kyoto-u.ac.jp
}

\begin{abstract}
Humans generate bipedal walking by cooperatively manipulating their complicated and redundant musculoskeletal systems to produce adaptive behaviors in diverse environments. To elucidate the mechanisms that generate adaptive human bipedal locomotion, we conduct numerical simulations based on a musculoskeletal model and a locomotor controller constructed from anatomical and physiological findings. In particular, we focus on the adaptive mechanism using phase resetting based on the foot contact information that modulates the walking behavior. For that purpose, we first reconstruct walking behavior from the measured kinematic data. Next, we examine the roles of phase resetting on the generation of stable locomotion by disturbing the walking model. Our results indicate that phase resetting increases the robustness of the walking behavior against perturbations, suggesting that this mechanism contributes to the generation of adaptive human bipedal locomotion.
\end{abstract}

keywords: musculoskeletal model, adaptability, foot-contact information, numerical simulation, central pattern generator $(\mathrm{CPG})$.

\section{Introduction}

Humans generate various motions by cooperatively manipulating their complicated and redundant musculoskeletal systems to produce adaptive behaviors in diverse environments. Humans can achieve adaptive bipedal walking through the interaction among the body, brain, and environment.

To elucidate the mechanisms for generating human bipedal locomotion, many researchers have conducted numerical simulations using mathematical models of the musculoskeletal and nervous systems based on anatomical and physiological findings. Taga et al. [26-28] examined the mechanisms to produce walking behavior through global entrainment among the musculoskeletal and neural systems and the environment. Ogihara and Yamazaki [23] emulated human bipedal walking using an anatomically and 
physiologically based neuromusculoskeletal model. Hase and Yamazaki [15] simulated the acquisition process of bipedal walking with a neuromusculoskeletal using genetic algorithms. Tomita and Yano [30] constructed basal ganglia and brainstem models and investigated the contributions of muscle tone and rhythm controls. Jo and Massaquoi [19] integrated the feedforward and feedback commands that drive the musculoskeletal model and incorporated movement and posture controls. Günther and Ruder [14] verified the $\lambda$-model of the muscle control mechanism in the generation of walking behavior.

In this paper, we focused on an adaptive locomotor mechanism using rhythm and phase modulation based on phase resetting. Phase resetting provides phase modification of rhythmic behaviors, which is observed in many biological systems and contributes to the entrainment behavior of oscillatory elements [18]. Yamasaki et al. [36,37] examined the roles of phase resetting in generating adaptive human bipedal locomotion against a force perturbation. They prepared joint angles during walking that were encoded by an oscillator phase and modulated the joint motions by resetting the oscillator phase when the walking model was disturbed. Their simulation illustrated that such phase reset prevents falling over against postural disturbances induced by the perturbations. Although they reset the phase when the walking model was disturbed, we investigated the contribution of phase resetting based on foot contact information in the following procedure. First, we dynamically reconstructed the walking behavior from the measured kinematic data by constructing a musculoskeletal model and a locomotor controller. We compared the simulation results with such measured data of the actual walking motion as the ground reaction forces and muscular electromyogram (EMG) activities that explain the dynamical contributions to locomotion and verified the simulation validity. After that, we examined the mechanism for generating adaptive walking behavior based on the constructed model. In particular, we investigated the roles of phase resetting using a force perturbation.

This paper is organized as follows: Section 2 introduces the musculoskeletal model, Section 3 explains the locomotor controller that drives the musculoskeletal model, and Section 4 investigates the walking behavior by numerical simulations based on the constructed model. Section 5 describes the discussion and conclusion.

\section{Musculoskeletal Model}

\subsection{Skeletal model}

Figure 1 shows the musculoskeletal model constructed as a two-dimensional model. The skeletal system consists of seven rigid links that represent the head, arms, and torso (HAT), thighs, shanks, and feet, as shown in Fig. 1A. We used the physical parameters of the skeletal model in [23]. The length of the sole, i.e., the distance between the heel and the head of the 5 th metatarsal, is $0.19 \mathrm{~m}$. Each joint is modeled as a pin joint and has a linear viscous element, and the coefficients of viscosity for the hip, knee, and ankle joints are 1.09, 3.17, and $0.943 \mathrm{Nms} / \mathrm{rad}$, respectively [8]. The joint angles become zero when the model stands straight; that is, the torso, thigh, and shank are in a straight line and perpendicular to 

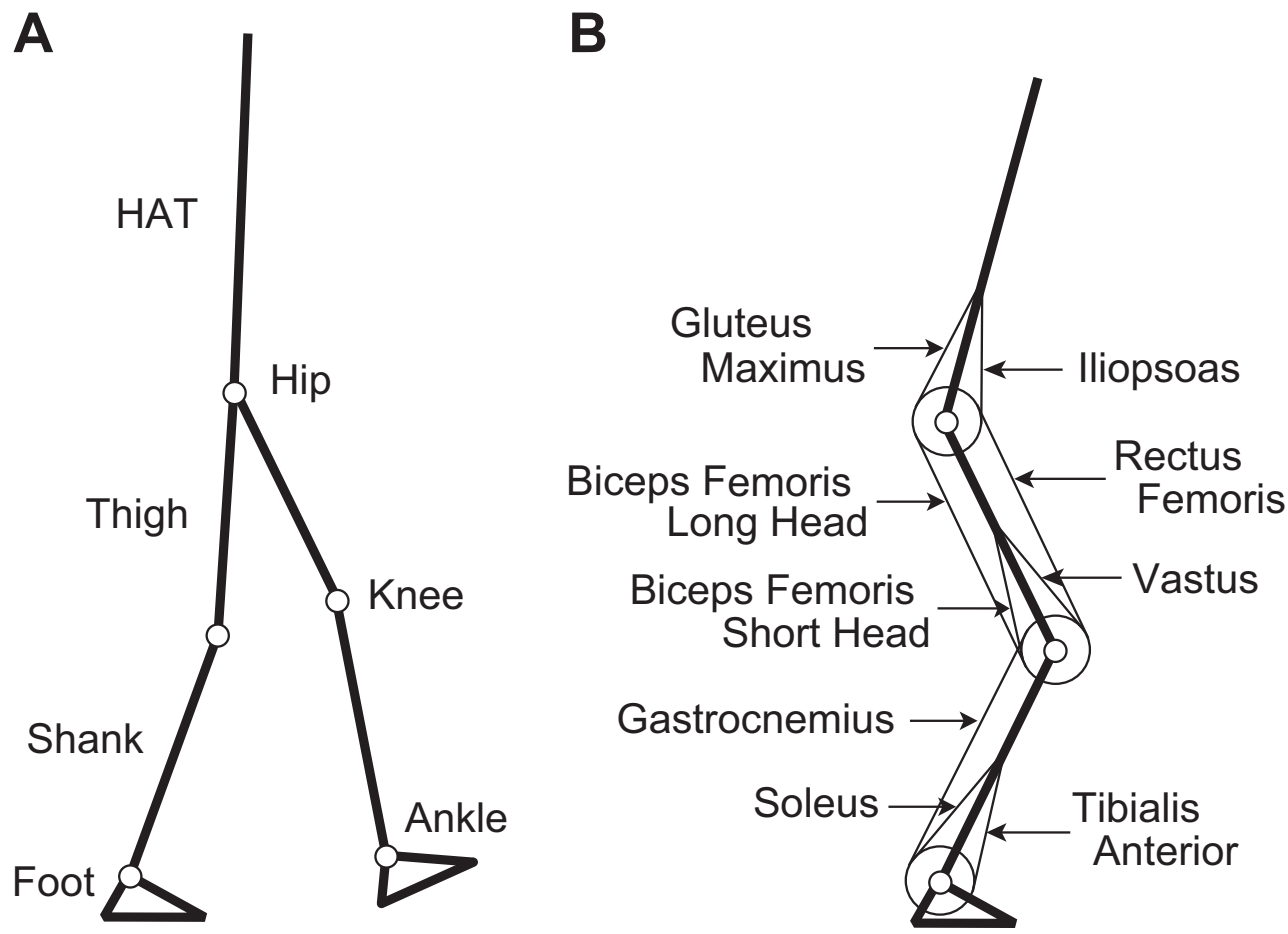

Figure 1: Schematic diagrams of musculoskeletal model [23]. A shows skeletal model composed of seven rigid links that represent head, arms, and torso (HAT), thighs, shanks, and feet. B displays muscular model for one leg composed of nine principal muscles; six muscles (IL, GM, VA, BFS, TA, and SO) are uniarticular and three (RF, BFL, and GC) are biarticular.

the sole of the foot. When a link rotates in an anticlockwise direction relative to the proximal link, the joint angle increases. When the foot contacts the ground, it receives the reaction force from the ground. We employed four contact points on the sole that receive the reaction force: toe, heel, and $4 \mathrm{~cm}$ inside from the toe and heel. The reaction force is modeled by a linear spring and damper system. The elastic and viscous coefficients are $5.0 \times 10^{3} \mathrm{~N} / \mathrm{m}$ and $1.0 \times 10^{2} \mathrm{Ns} / \mathrm{m}$ horizontally and $2.5 \times 10^{4} \mathrm{~N} / \mathrm{m}$ and $5.0 \times 10^{2} \mathrm{Ns} / \mathrm{m}$ vertically.

\subsection{Muscular model}

We incorporated nine principal muscles for each leg, as shown in Fig. 1B; six muscles act over single joints: hip flexion (Iliopsoas (IL)), hip extension (Gluteus Maximus (GM)), knee extension (Vastus $(\mathrm{VA}))$, knee flexion (Biceps Femoris Short Head (BFS)) , ankle flexion (Tibialis Anterior (TA)), and ankle extension (Soleus $(\mathrm{SO})$ ), and three muscles are bifunctional: hip flexion and knee extension (Rectus Femoris (RF)), hip extension and knee flexion (Biceps Femoris Long Head (BFL)) , and knee flexion and ankle extension (Gastrocnemius (GC)). The moment arms of the muscles around the joints are assumed to be constant regardless of the joint angles. This yields that muscle length $L_{m}$ of muscle 
$m(m=\mathrm{IL}, \mathrm{GM}, \mathrm{VA}, \mathrm{BFS}, \mathrm{TA}, \mathrm{SO}, \mathrm{RF}, \mathrm{BFL}$, and GC) is derived from

$$
\begin{aligned}
L_{m} & =\bar{L}_{m}+\sum_{j=\left\{j_{m}\right\}} \operatorname{sign}_{m} M_{m}^{j}\left(\theta^{j}-\bar{\theta}^{j}\right) \\
\operatorname{sign}_{m} & = \begin{cases}-1 & m=\mathrm{IL}, \mathrm{VA}, \mathrm{TA}, \mathrm{RF} \\
+1 & m=\mathrm{GM}, \mathrm{BFS}, \mathrm{SO}, \mathrm{BFL}, \mathrm{GC}\end{cases}
\end{aligned}
$$

where $\bar{L}_{m}$ is the muscle optimal length, $M_{m}^{j}$ is the moment arm for joint $j$ ( $j=$ hip, knee, ankle), $\theta^{j}$ is the joint angle, $\bar{\theta}^{j}$ is the neutral joint angles, and $\left\{j_{m}\right\}$ indicates the corresponding joints, e.g., $\left\{j_{\mathrm{GC}}\right\}=\{$ knee, ankle $\}$. When the joints are at their neutral joint angles, the muscles are at their muscle optimal lengths. Such neutral joint angles are assumed to be 0.0, -0.52, and 0.0 rad for the hip, knee, and ankle joints, respectively [23].

A muscle receives a signal from the corresponding $\alpha$-motoneuron and generates muscle tension depending on the force-length and force-velocity relationships. We used the mathematical model in [23], composed of contractile element $(\mathrm{CE})$ and passive elastic $(\mathrm{PE})$ and damping $(\mathrm{PD})$ elements parallel to CE, as follows:

$$
\begin{aligned}
& F_{m}=\bar{F}_{m}^{C E} \cdot k\left(\xi_{m}\right) \cdot h\left(\eta_{m}\right) \cdot a_{m}+F_{m}^{P D}+F_{m}^{P E} \\
& k\left(\xi_{m}\right)=0.32+0.71 \exp \left[-1.112\left(\xi_{m}-1\right)\right] \sin \left[3.722\left(\xi_{m}-0.656\right)\right] \\
& h\left(\eta_{m}\right)=1+\tanh \left(3.0 \eta_{m}\right) \\
& F_{m}^{P D}=c_{m}^{P D} \dot{L}_{m} \\
& F_{m}^{P E}=k_{m}^{P E}\left\{\exp \left[15\left(L_{m}-\bar{L}_{m}\right)\right]-1\right\}
\end{aligned}
$$

where $F_{m}$ is the muscle tension, $\bar{F}_{m}^{C E}$ is the maximum muscle tension by CE, $k\left(\xi_{m}\right)$ is the force-length relationship, $h\left(\eta_{m}\right)$ is the force-velocity relationship, $\xi_{m}$ and $\eta_{m}$ are the normalized muscle length and contractile velocity divided by the muscle optimal length and maximum muscle contractile velocity $\overline{\dot{L}}_{m}$ (= $3.0 \mathrm{~m} / \mathrm{s}$ for all muscles); that is, $\xi_{m}=L_{m} / \bar{L}_{m}$ and $\eta_{m}=\dot{L}_{m} / \overline{\dot{L}}_{m}, a_{m}$ is the muscle activation induced by the signal from the corresponding $\alpha$-motoneuron $\left(0 \leq a_{m} \leq 1\right), F_{m}^{P D}$ and $F_{m}^{P E}$ are the forces generated by the damping and elastic elements, $c_{m}^{P D}$ is the viscous coefficient, and $k_{m}^{P E}$ is the coefficient of the elastic element. We used the physical parameters of the muscular model in [23]. Muscle torque $\tau^{j}$ of joint $j$ is obtained by summing the contributions from all muscles that act at the joint and given by

$$
\tau^{j}=\sum_{m=\left\{m_{j}\right\}}-\operatorname{sign}_{m} M_{m}^{j} F_{m}
$$

where $\left\{m_{j}\right\}$ indicates the corresponding muscles, e.g., $\left\{m_{\text {knee }}\right\}=\{\mathrm{VA}, \mathrm{BFS}, \mathrm{RF}, \mathrm{BFL}, \mathrm{GC}\}$. 


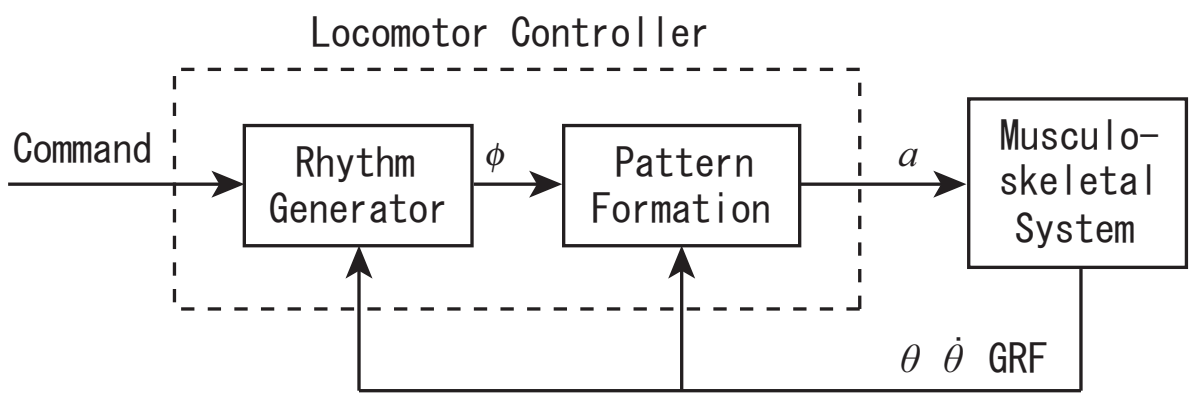

Figure 2: Block diagram of simulation model

\section{Locomotor Controller}

\subsection{Locomotor CPG}

Motoneurons that control the activity of muscles receive signals from Central Pattern Generators (CPGs) as well as signals from various sensory organs. CPGs can produce oscillatory behaviors in the absence of rhythmic input and proprioceptive feedback. Physiological findings imply that CPGs consist of hierarchical networks through interneurons: Rhythm Generator (RG) and Pattern Formation (PF) networks $[6,25]$. The RG network generates the basic rhythm and alters it by producing phase shift and rhythm resetting affected by sensory afferents and perturbations. The PF network shapes the rhythm into spatiotemporal patterns of the activation of motoneurons through interneurons.

\subsection{Locomotor controller using phase oscillators}

Figure 2 shows the block diagram of our simulation model that consists of Rhythm Generator (RG) and Pattern Formation (PF) blocks, both of which receive commands descending from higher centers, joint state information, and foot contact information (ground reaction force (GRF)) to create motor commands. For the RG block we used two simple phase oscillators, each of which produces the basic rhythm and phase information for the corresponding leg motion, since the rhythmic behavior of a pair of flexor and extensor muscles during walking can be represented by one oscillator [25]. Let $\phi$ be the oscillator phase for one leg and $\psi$ be the oscillator phase for the other leg $(0 \leq \phi, \psi \leq 2 \pi)$, and then the oscillator phases follow the dynamics

$$
\begin{aligned}
\dot{\phi} & =\omega-K_{\Delta} \sin (\phi-\psi-\pi) \\
\dot{\psi} & =\omega-K_{\Delta} \sin (\psi-\phi-\pi)
\end{aligned}
$$

where $\omega$ is a parameter that produces the basic rhythm and $K_{\Delta}$ is the gain parameter. The second term of the right-hand side indicates a function that maintains the interlimb coordination so that the legs move out of phase. We assumed that the commands from the higher centers contain the basic rhythm $(\omega)$ of the locomotor behavior and used the same value for $\omega$ and $K_{\Delta}$ for both legs. We used $\omega=2 \pi$ to generate the walking motion of a gait cycle of $1.0 \mathrm{~s}$ as described below and $K_{\Delta}=1.0$. 
To dynamically reconstruct walking behavior from the measured kinematic data, we modeled the PF block using PD feedback control. Specifically, we used the two-dimensional position data of markers attached to the hip, ankle, toe, and heel during walking in [33], whose gait cycle is about $1.0 \mathrm{~s}$. We calculated the joint kinematics by adapting the position data to the skeletal model in Sec. 2.1, and from Eq. (1) we achieved desired length $L_{m}^{*}$ of each muscle for one gait cycle. To express periodicity, the desired length is encoded using the phase of the corresponding oscillator as $L_{m}^{*}(\phi)$. It is then normalized by the muscle optimal length and used as nominal length $\xi_{m}^{*}(\phi)$. To establish the desired motion at individual muscles, we determined muscle activation $a_{m}$ by

$$
a_{m}=\kappa\left(\xi_{m}-\xi_{m}^{*}(\phi)\right)+\sigma \eta_{m}
$$

where $\kappa$ and $\sigma$ are gain parameter using the same value between the muscles. When $a_{m}<0$ we set $a_{m}=0$, and when $a_{m}>1$ we put $a_{m}=1$. This model aims to determine the desired joint configuration and muscle state based on the phase information from the RG block and then to produce the signals that activate the muscles.

We used gain parameters $\kappa=190$ and $\sigma=8$ with the following physical characteristics. Suppose that the musculoskeletal model stands straight while both soles contact the ground. The ankle torque can produce muscle torque to control the balance by muscle contractile activity whose proportional gain of the ankle joint corresponds to $1.9 \mathrm{Mgh}$ for the direction of flection and $7.4 \mathrm{Mgh}$ for the direction of extension, where $M$ is the body weight, $g$ is the acceleration of gravity, and $h$ is the distance between the ankle and the center of mass. These gains are obtained by summing value $\kappa\left(M_{m}^{\text {ankle }}\right)^{2} \bar{F}_{m}^{C E} / \bar{L}_{m}$ of the corresponding muscles calculated from Eqs. (1), (2), (3), and (5). When the model is supported by one leg, the gains become $0.95 \mathrm{Mgh}$ and $3.7 \mathrm{Mgh}$ for the direction of flection and extension, respectively, implying that the gain parameters are not so large that the posture is always stabilized by the muscle activities.

In general, posture control is crucial to modulate walking behavior based on somatosensory information. However, since we generate walking motion from the measured kinematic data, the locomotor controller implicitly contains posture control. Therefore, we don't explicitly incorporate a posture control scheme.

\subsection{Phase resetting based on foot contact information}

In this section, we incorporated the adaptive mechanism to modulate the walking behavior based on sensory information. As mentioned in Sec. 3.1, the RG network creates the basic rhythm, and the PF network produces patterns of signals based on the rhythm information from the RG network, which modulates the basic rhythm by producing phase shift and rhythm resetting affected by sensory afferents and perturbations. Since the PF network produces signal patterns based on the rhythm information from the RG network, such modulation of the basic rhythm affects signal generation in the PF network and seems to play an important role in generating adaptive walking behavior. 
We incorporated the phase and rhythm modulation based on phase resetting using the sensory information about the phase transition from the swing to stance phase and vice versa. Let $\phi_{\text {cont }}$ be the oscillator phase when the leg motion changes from the swing to the stance phase and $\phi_{\text {off }}$ be the oscillator phase when the leg motion alters from the stance to the swing phase. When the foot lands on the ground and receives the ground reaction force, we reset the oscillator phase of the leg to $\phi_{\text {cont }}$. When the foot leaves the ground and the ground reaction force vanishes, we reset its oscillator phase to $\phi_{\text {off }}$. However, note that the discrete change of the oscillator phase induces discrete changes in the desired motions, influencing the simulation results since the locomotor controller creates command signals based on the PD feedback control. To deal with such discrete changes and to adequately incorporate phase resetting, we modified the oscillator phase dynamics (4) as follows. First, we prepared alternative oscillator phases $\hat{\phi}$ for one leg and $\hat{\psi}$ for the other leg $(0 \leq \hat{\phi}, \hat{\psi} \leq 2 \pi)$. These oscillator phases basically follow phase dynamics (4) and are reset based on the foot contact information. The phase dynamics for these alternative oscillators are written by

$$
\begin{aligned}
& \dot{\hat{\phi}}=\omega-K_{\Delta} \sin (\hat{\phi}-\hat{\psi}-\pi)-\left(\hat{\phi}-\phi_{\text {cont }}\right) \delta\left(t-t_{\text {cont }}^{\phi}\right)-\left(\hat{\phi}-\phi_{\text {off }}\right) \delta\left(t-t_{\text {off }}^{\phi}\right) \\
& \dot{\hat{\psi}}=\omega-K_{\Delta} \sin (\hat{\psi}-\hat{\phi}-\pi)-\left(\hat{\psi}-\phi_{\text {cont }}\right) \delta\left(t-t_{\text {cont }}^{\psi}\right)-\left(\hat{\psi}-\phi_{\text {off }}\right) \delta\left(t-t_{\text {off }}^{\psi}\right)
\end{aligned}
$$

where $\delta(\cdot)$ is Dirac's delta function, $t_{\text {cont }}^{j}(j=\phi, \psi)$ is the time when the foot of the corresponding leg lands on the ground, and $t_{\text {off }}^{j}(j=\phi, \psi)$ is the time when the foot of the corresponding leg leaves the ground. The third and fourth terms of the right-hand sides are induced by phase resetting, which causes discrete changes of the alternative oscillator phases. To make oscillator phases $\phi$ and $\psi$ continuously catch up with these alternative oscillator phases $\hat{\phi}$ and $\hat{\psi}$, we modified phase dynamics (4) into

$$
\begin{aligned}
\dot{\phi} & =\omega-K_{\Delta} \sin (\phi-\psi-\pi)-K_{\hat{\Delta}}(\phi-\hat{\phi}) \\
\dot{\psi} & =\omega-K_{\Delta} \sin (\psi-\phi-\pi)-K_{\hat{\Delta}}(\psi-\hat{\psi})
\end{aligned}
$$

where $K_{\hat{\Delta}}$ is the gain parameter. We used $K_{\hat{\Delta}}=10$.

\section{Simulation Results}

\subsection{Dynamical reconstruction of locomotion from measured kinematic data}

For the first approach, we reconstructed the walking behavior from the measured kinematic data using the mathematical models without incorporating the phase resetting mechanism.

Figure 3 shows the simulated walking behavior illustrated by the stick diagram where the display interval is $0.1 \mathrm{~s}$. Figure 4 compares the simulation results and the measured data of the actual walking motion. Although it only shows the trajectories for one cycle, the trajectories of the simulation results are identical over the step cycles after calculation of several seconds, meaning that the model achieved stable walking behavior. A shows the joint kinematics for one leg, verifying that the numerical simulation kinematically reconstructed the measured data. $\mathrm{HC}$ and $\mathrm{TO}$ indicate the heel contact and toe off of 


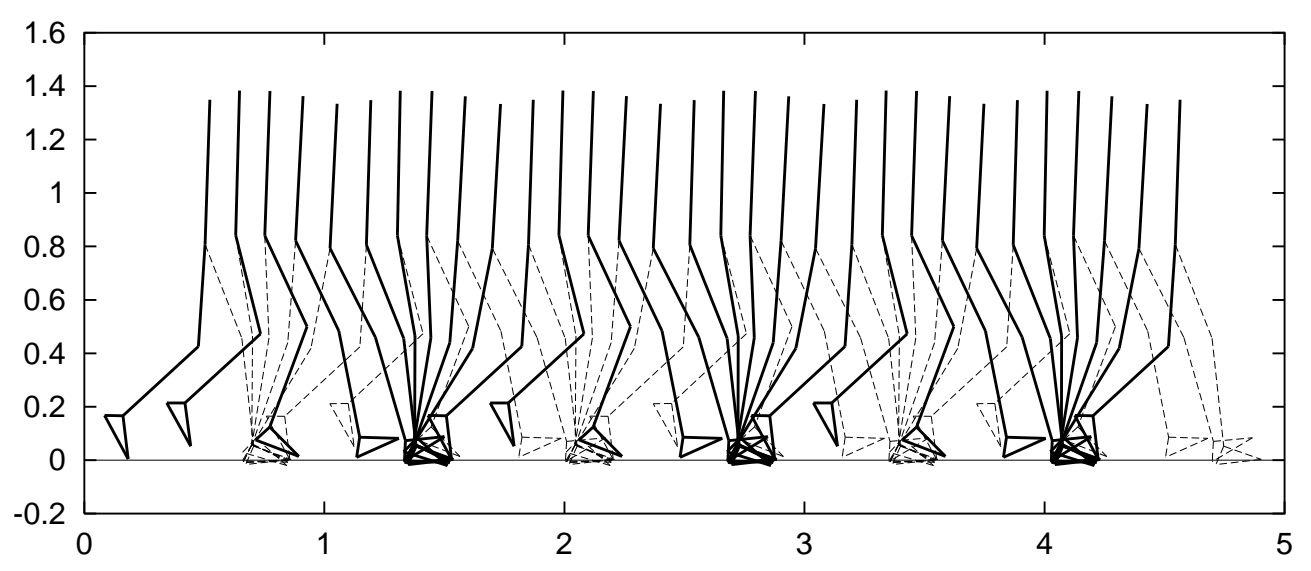

Figure 3: Stick diagram of simulated bipedal locomotion

the corresponding leg in the simulated walking behavior. The duty factor of the simulated walking motion is 0.59. B displays the vertical and horizontal ground reaction forces, where the measured data are used in [33]. As shown in the vertical reaction force of the measured data, the double-peaked force curve is a characteristic property in human bipedal walking. Although the simulation result has almost three peaks and possesses discrete responses due to the foot contact, it established a similar shape to the measured data. Regarding the horizontal reaction force, the simulation result achieved breaking and propulsive forces at the beginning and end of the stance phase, and its profile resembles the measured data. $\mathbf{C}$ shows the muscle activation patterns of the nine muscles compared with the measured EMG data in [17], where the solid lines are the simulated results and the filled gray areas are the measured EMG data. Although the accuracy of the dynamical reconstruction of the muscle activity is different between the muscles, the simulation results established the characteristic features of the muscle activation patterns. Muscle IL obtained the peak of the muscle activities at the beginning of the swing phase. Muscle GM achieved muscle activity from the end of the swing phase to the beginning of the stance phase. Muscle BFS produced muscle activities at the beginning and end of the swing phase. Muscle TA obtained muscle activities at the beginning of the stance phase and during the swing phase. Muscles SO and GC achieved synchronous activity at the end of the stance phase. This comparison reflects that numerical simulation reconstructed the dynamical walking behavior from kinematical data with similar characteristics to the measured data. 


\section{A. Joint angles}

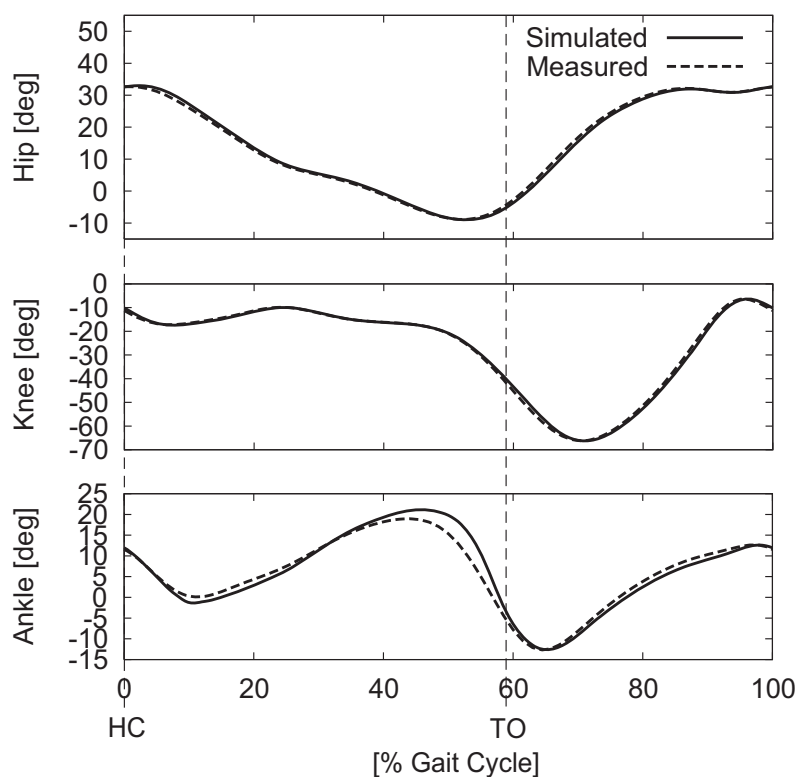

B. Ground reaction forces

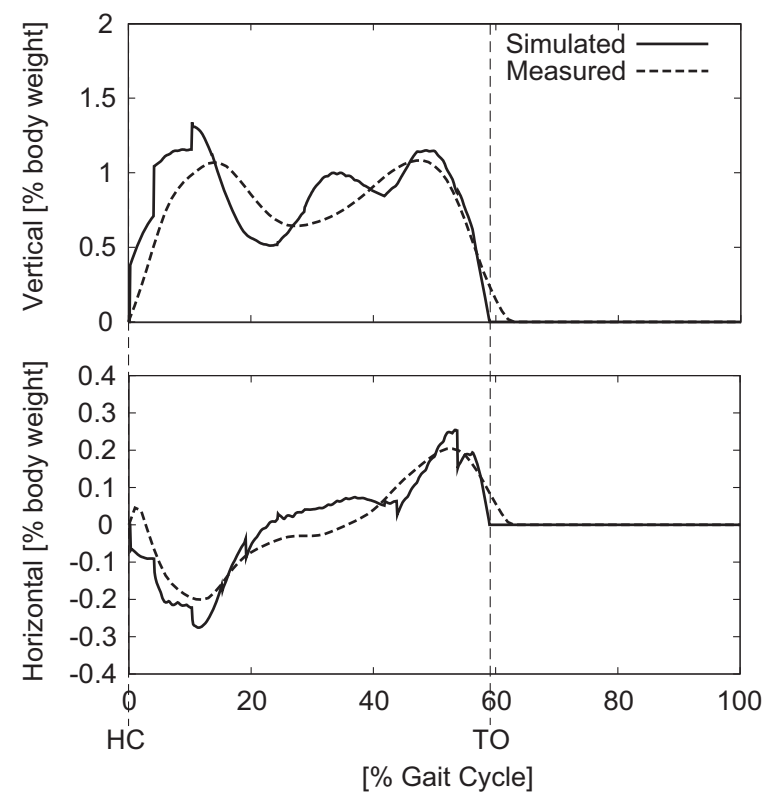

\section{Muscle activation patterns}

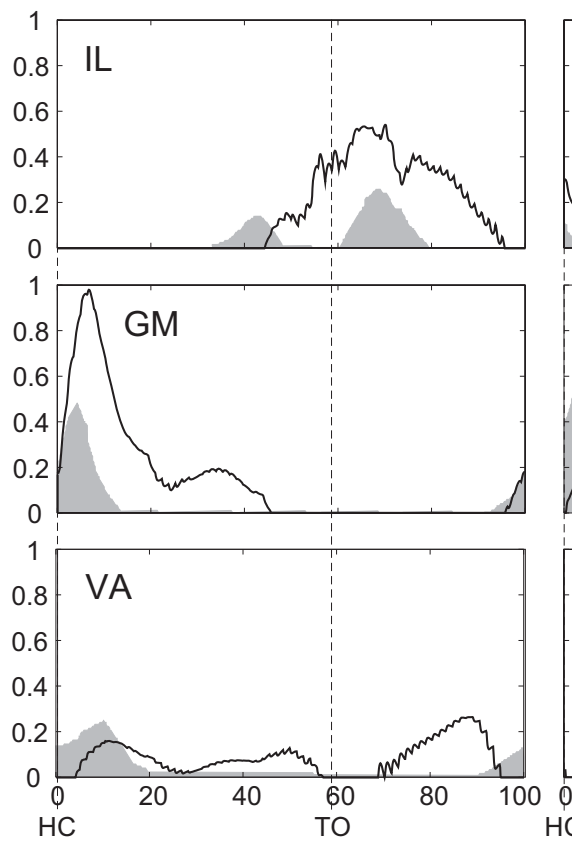

[\% Gait Cycle]

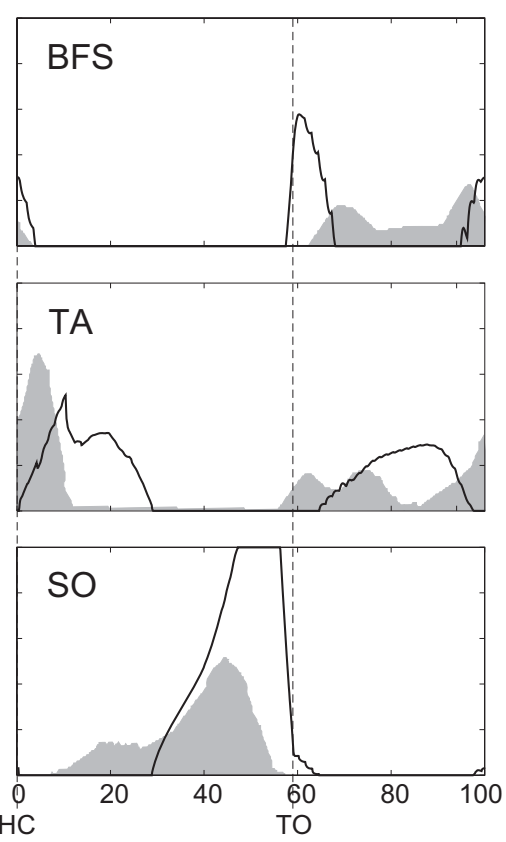

[\% Gait Cycle]

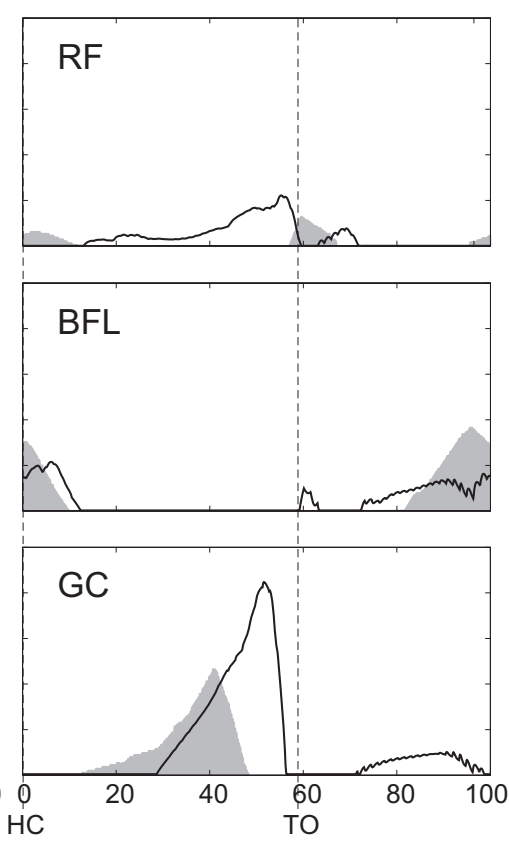

[\% Gait Cycle]

Figure 4: Comparisons between simulation results and measured data of actual walking motion. A, B, and $\mathbf{C}$ show joint angles, ground reaction forces, and muscle activation patterns, respectively. Measured data of joint angles and ground reaction forces are used in [33], and muscle activation patterns were compared with measured EMG data in [17]. In C, solid lines are simulated results and filled gray areas are measured EMG data. HC and TO indicate heel contact and toe off of simulation results. 


\subsection{Adaptive walking by phase resetting}

The resultant duty factor of the simulated walking behavior obtained in the last section was 0.59 , as shown in Fig. 4. Therefore, $\phi_{\text {cont }}$ is set to $0(2 \pi)$ and $\phi_{\text {off }}$ is set to $0.59 \times 2 \pi$. To examine the roles of the phase resetting at foot-contact and foot-off, we compared four cases: without phase reset, using phase reset at foot-contact, using phase reset at foot-off, and using phase reset at both foot-contact and foot-off. In particular, we investigated the adaptability against force disturbances. Specifically, after the walking model achieved steady walking behavior, we added a force perturbation for $0.1 \mathrm{~s}$ to the center of mass of HAT in the horizontal direction (forward or backward) and examined whether the model keeps walking or falls down; we changed the magnitude and timing of the perturbation and determined that the model keeps walking when it doesn't fall down over $30 \mathrm{~s}$ after being disturbed.

Figure 5 shows the simulation results, where the upper and lower boundaries indicate the limitations of the perturbations added in the forward and backward directions, respectively, where the model keeps walking without falling over. By comparing with the case without phase reset, the model walked adaptively to the perturbation in the backward direction using phase reset at foot-contact. On the contrary, it established adaptive walking against perturbation in the forward direction using phase reset at foot-off. Using phase reset at both foot-contact and foot-off, it walked adaptively to the perturbation in both directions. Figure 6 shows the response to force perturbation using phase reset at both footcontact and foot-off. The upper figures display simulated walking behaviors and the lower figures illustrate the timings of the foot-contact of the right leg, and the vertical bars indicate the timings. A shows walking behavior without perturbation. B adds perturbation of $-140 \mathrm{~N}$ at a $30 \%$ gait cycle. When the translational speed of HAT decreases, it slightly tilts backward. Therefore, foot-contact is delayed after being disturbed. However, walking behavior recovered soon. C gives the perturbation of $50 \mathrm{~N}$ at a $30 \%$ gait cycle. The translational speed of HAT increases and it tilts forward. Therefore, foot-contact advances just after being disturbed, but after the second foot-contact, it is delayed. However, again, walking behavior recovered soon. 


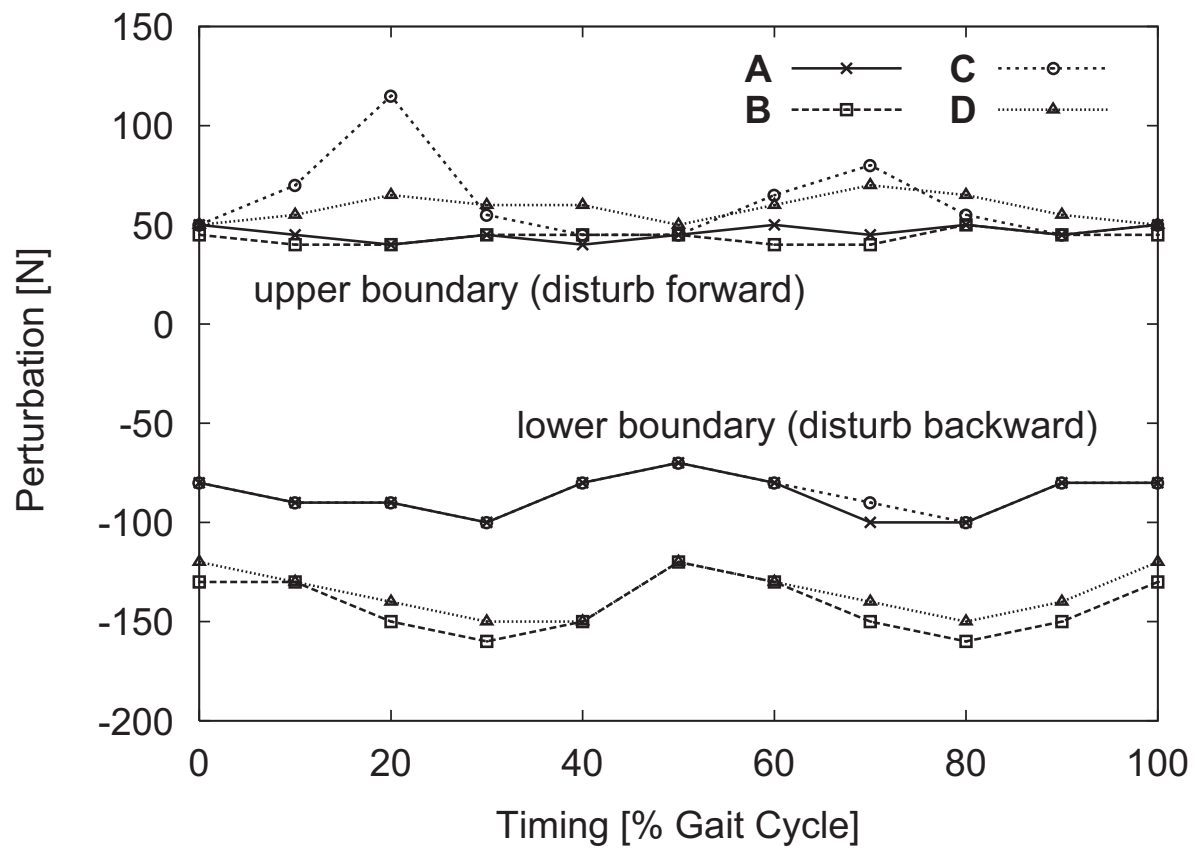

Figure 5: Limitation to keep walking against force perturbation. A: without phase reset. B: using phase reset at foot-contact. C: using phase reset at foot-off. $\mathbf{D}$ : using phase reset at foot-contact and foot-off. 
A

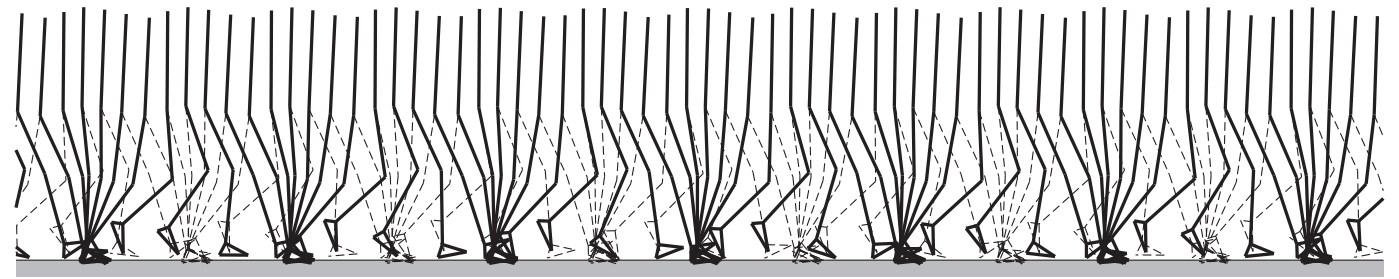

B

:
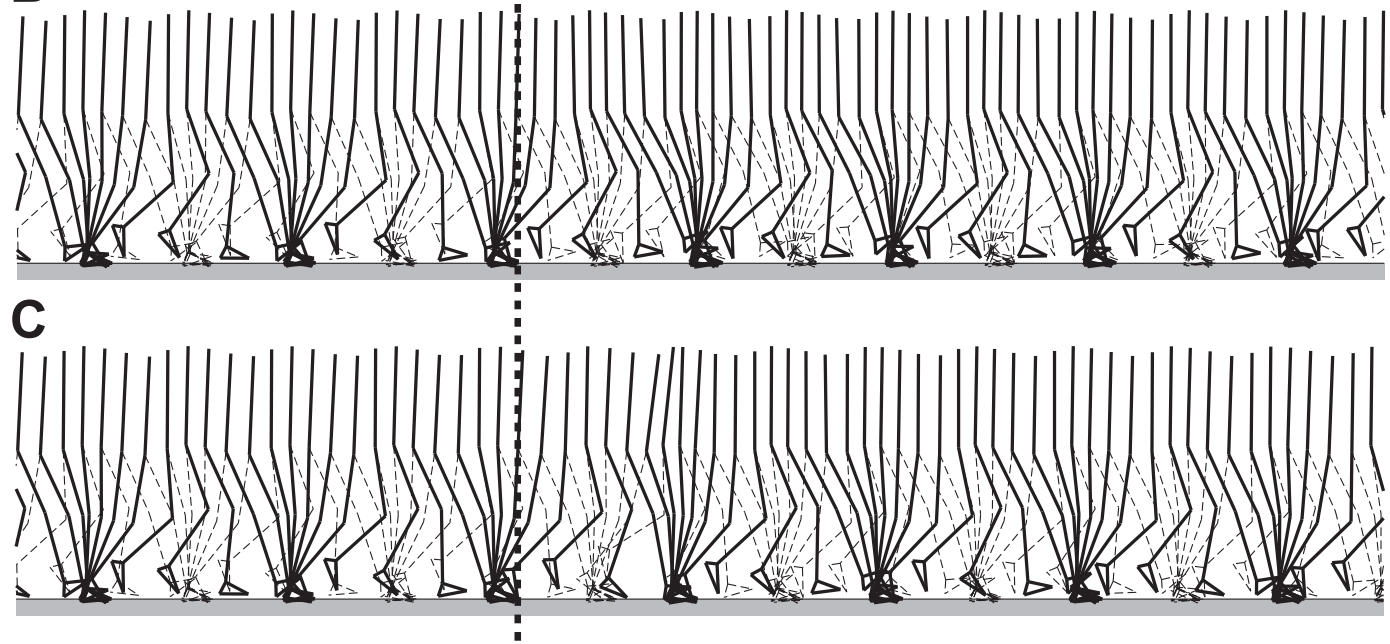

perturbation
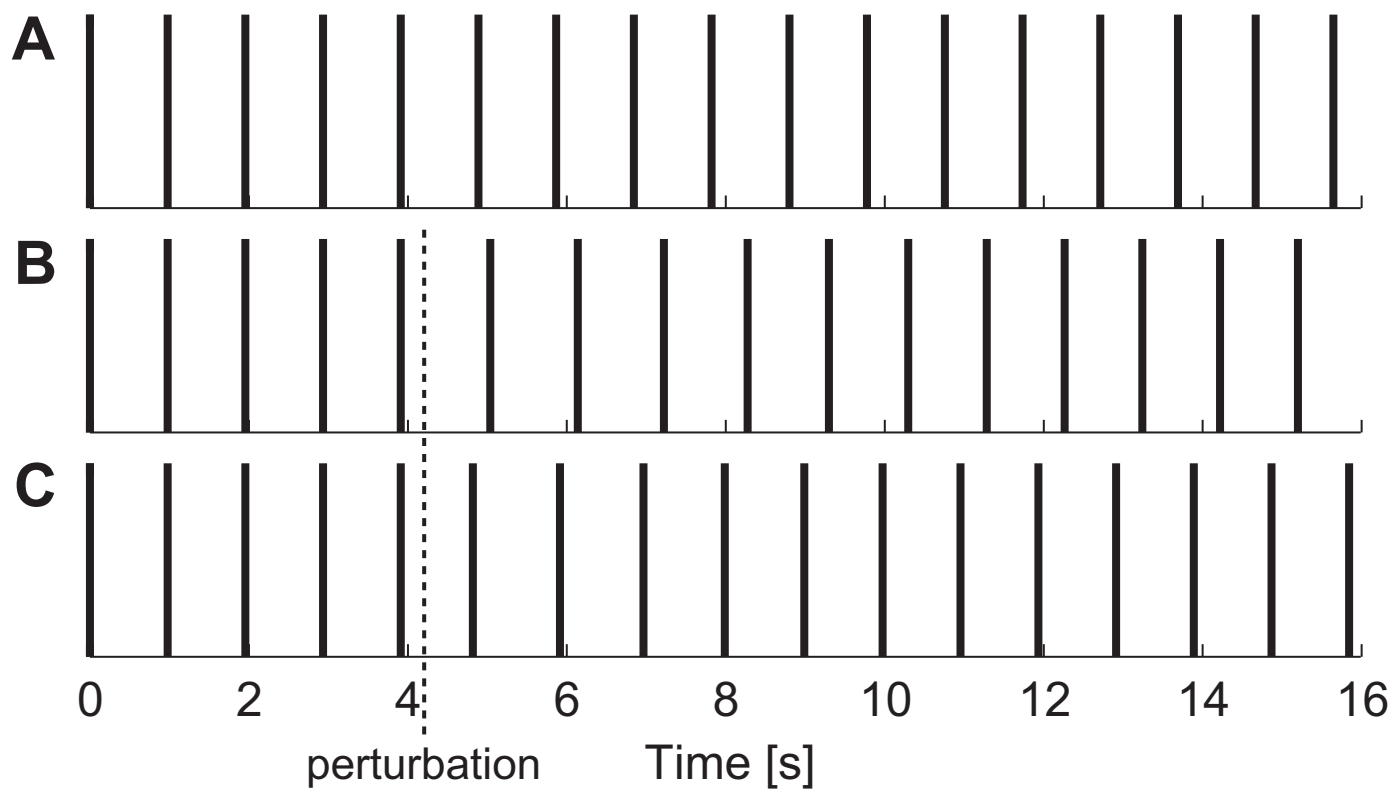

Figure 6: Response to force perturbation using phase reset at both foot-contact and foot-off. Upper figures show simulated walking behaviors and lower figures illustrate timings of foot-contact of right leg (vertical bars). A: without perturbation. B: $-140 \mathrm{~N}$ at $30 \%$ of gait cycle. C: $60 \mathrm{~N}$ at $30 \%$ of gait cycle. 


\section{Discussion}

The contribution of this paper mainly consists of two parts. One is the dynamical reconstruction of walking behavior from the measured kinematic data of actual human walking motion based on the musculoskeletal model and locomotor controller. The other is an investigation of phase resetting to achieve adaptive walking.

\subsection{Dynamical reconstruction of walking behavior}

In this paper, we produced bipedal walking through numerical simulations from the measured kinematic data of actual human bipedal walking. Our results established dynamically stable locomotor behavior that is comparable with such measured data during actual human walking as ground reaction forces and muscular EMG activities. Such forward dynamic simulation is useful to estimate the internal states of musculoskeletal systems. In addition, applications to the investigation of animal behaviors, whose internal states are more difficult to measure than humans and which possess much adaptability, might offer great insight into the elucidation of the mechanisms inherent in adaptive locomotor behaviors [24].

Many previous studies have investigated human bipedal walking based on inverse dynamics and optimization using measured data $[1,2,8]$. Although these approaches are useful to examine the dynamical contribution to generate walking behavior, it is difficult to treat walking stability; that is, to examine if the walker can keep walking or will fall down over time using the calculated results. However, forward dynamic simulation can deal with walking stability, which enables discussion about such functional roles as adaptability against disturbances.

In the forward dynamic simulation of locomotor behavior, the determination of the parameters in the controller is generally crucial. Since we constructed a locomotor controller based on PD feedback control to reproduce the measured kinematic data, gain parameters $\kappa$ and $\sigma$ in Eq. (5) are the most crucial. When these parameters are small, it is difficult to establish desired joint motions and stable locomotor behavior. On the other hand, when they are sufficiently large, even though the joint motions will easily achieve the desired behaviors, they react immoderately to disturbances and the simulation may yield unrealistic results that are different from the measured data. Therefore, the gain parameters must be adequately determined for the present simulation of human walking.

\subsection{Investigation of roles of phase resetting}

We investigated the adaptability of locomotor behavior by focusing on phase resetting that modulates walking behavior based on sensory information. In particular, we concentrated on such foot contact information as foot-contact and foot-off, because they represent the dynamical interactions between the body and environment. We examined the roles of phase resetting by adding a force perturbation during walking.

The present study demonstrated that phase resetting based on foot contact information does actually improve the robustness of human bipedal walking. The walking model was better capable of coping with 
larger force perturbation during walking when it incorporated the phase resetting mechanism. Furthermore, our simulation suggests that phase resetting at foot-off and foot-contact was more responsible for force perturbation in the forward and backward force perturbation, as shown in Fig. 5. We also found that phase resetting seems more effective against force perturbation in the backward direction. The intermittent modulation of the walking phase based on foot contact information therefore seems to be a powerful mechanism for generating robust human locomotion.

Physiological studies to date have indicated that such phase resetting or rhythm modulation based on foot contact information actually exists in animals and contributes to the successful generation of stable periodic locomotion. For example, cats are known to use two types of sensory information to change the phase from stance to swing: force-sensitive afferents $[9,32]$ and position-sensitive afferents $[13,16]$. Ekeberg and Pearson [10] carried out numerical simulations of the locomotor behavior of cats with a musculoskeletal model of the hind limbs to investigate the roles of sensory information, suggesting that the phase transition induced by force information makes a larger contribution to generate adaptive behavior. Yakovenko et al. [35] conducted numerical simulations of cat locomotor behavior, where they prepared the patterns of signals for the swing and stance phases delivered to motoneurons from the measured EMG data and switched the phases based on joint configuration and ground reaction forces. Their simulation suggests that such phase switch between the swing and stance, and phase shift and resetting, depending on afferent feedback modulate the signal generation and the walking period, which plays important roles in establishing adaptive walking. Our simulation suggests that similar mechanisms might exist in human locomotor control and contribute to the generation of adaptive locomotor behavior.

Although in our simulation the oscillator phase values to be reset based on foot-contact information are predetermined, the amount of phase resetting depends on the states of the walking model. Since phase resetting occurs every gait cycle, perturbation in one direction doesn't mean that the phase is reset in one direction (gait cycle is lengthened or shortened), as shown in Fig. 6C. When the foot lands on the ground or it leaves the ground, only the phase of the corresponding leg is reset, which fluctuates the interlimb coordination. However, the interactions in the phase dynamics between the oscillator phases maintain them out of phase.

The rhythm and phase modulation due to phase resetting and interactions seems to contribute to posture control, although our model doesn't explicitly incorporate it. Such dynamical contributions must be investigated in detail to clarify the adaptive mechanism. Although we used motor commands and proprioceptive and sensory information, we didn't incorporate signal transmission delay. Since we determined muscle activations using PD feedback control, such delay might easily destabilize the walking behavior and limit the feedback gains. Furthermore, we basically prescribed the desired motions, although they were modulated through the phase resetting. Humans produce more skillful motions by cooperatively manipulating many degrees of freedom to establish adaptive walking. To create a more plausible model of human bipedal walking, we must take them into account. 


\subsection{Comparisons with biped robots}

In robotics research, many studies have concentrated on the generation, stabilization, and improvement of the efficiency of the walking behavior of biped robots. Many sophisticated biped robots have been developed that have successfully achieved walking behaviors. Most researchers have focused on modelbased approaches using motion planning based on zero moment point (ZMP) criterion [31]. To keep the robot from tumbling, they design joint desired trajectories so that ZMP remains within the convex hull of all contact points between the feet and ground. To easily design the controller under such dynamic constraints, the robot soles are basically flat on the ground while the feet contact the ground, which is different from human bipedal walking that has such dynamic foot actions as heel strike and toe off. The desired motion of our walking model was generated from the measured kinematic data, and we didn't consider whether the ZMP meets the dynamic condition. However, here the oscillator phases, which determine the desired motions, were modulated by phase resetting; hence adaptive walking behavior was established through the dynamical interactions between the musculoskeletal system, the locomotor controller, and the environment [26-28].

Inspired by humans and animals, many studies have constructed locomotion controllers by modeling the neural system, and simple biped robots established their walking behaviors $[11,12,20,21]$. However, it is not altogether clear how to create adaptive walking behavior through complicated musculoskeletal systems and various environments. The findings from simulation studies will contribute to designing controller and mechanical systems. Actually, many robotic studies have demonstrated the advantage of a phase resetting mechanism to achieve adaptive walking behavior [3-5, 21, 22]. In addition, as in phase resetting, biped robots inspired by passive dynamic walking were actuated by triggers based on foot-contact information and established energy efficient walking $[7,29,34]$. A common principle in the generation of walking behavior might exist in such phase resetting mechanisms among robots, humans, and animals.

\section{Acknowledgment}

This paper is supported in part by a Grant-in-Aid for Scientific Research on Priority Areas 'Emergence of Adaptive Motor Function through Interaction between Body, Brain and Environment' and a Grant-inAid for Young Scientists (B) (No. 19760173) from the Japanese Ministry of Education, Culture, Sports, Science and Technology and was also supported by Center of Excellence for Research and Education on Complex Functional Mechanical Systems (COE program of the Ministry of Education, Culture, Sports, Science and Technology, Japan).

\section{REFERENCES}

[1] F.C. Anderson and M.G. Pandy, Static and dynamic optimization solutions for gait are practically equivalent. J. Biomec., 34(2), 153-161 (2001). 
[2] F.C. Anderson and M.G. Pandy, Dynamic optimization of human walking, J. Biomech. Eng.. 123, 381-390 (2001).

[3] S. Aoi and K. Tsuchiya, Locomotion control of a biped robot using nonlinear oscillators. Autonomous Robots, 19(3), 219-232 (2005).

[4] S. Aoi and K. Tsuchiya, Stability analysis of a simple walking model driven by an oscillator with a phase reset using sensory feedback. IEEE Trans. Robotics, 22(2), 391-397 (2006).

[5] S. Aoi and K. Tsuchiya, Adaptive behavior in turning of an oscillator-driven biped robot. Autonomous Robots, 23(1), 37-57 (2007).

[6] R.E. Burke, A.M. Degtyarenko, and E.S. Simon, Patterns of locomotor drive to motoneurons and last-order interneurons: Clues to the structure of the CPG. J. Neurophysiol., 86, 447-462 (2001).

[7] S.H. Collins, A.L. Ruina, R. Tedrake, and M. Wisse, Efficient bipedal robots based on passivedynamic walkers. Science, 307, 1082-1085 (2005).

[8] D.T. Davy and M.L. Audu, A dynamic optimization technique for predicting muscle forces in the swing phase of gait. J. Biomech., 20(2), 187-201 (1987).

[9] J. Duysens and K.G. Pearson, Inhibition of flexor burst generation by loading ankle extensor muscles in walking cats. Brain Res., 187, 321-332 (1980).

[10] Ö. Ekeberg and K. Pearson, Computer simulation of stepping in the hind legs of the cat: An examination of mechanisms regulating the stance-to-swing transition. J. Neurophysiol., 94, 42564268 (2005).

[11] T. Geng, B. Porr, and F. Wörgötter, A reflexive neural network for dynamic biped walking control. Neural Comput., 18, 1156-1196 (2006).

[12] T. Geng, B. Porr, and F. Wörgötter, Fast biped walking with a sensor-driven neuronal controller and real-time online learning. Int. J. Robot. Res., 25(3), 243-259 (2006).

[13] S. Grillner and S. Rossignol, On the initiation of the swing phase of locomotion in chronic spinal cats. Brain Res., 146, 269-277 (1978).

[14] M. Günther and H. Ruder, Synthesis of two-dimensional human walking: a test of the $\lambda$-model. Biol. Cybern., 89, 89-106 (2003).

[15] K. Hase and N. Yamazaki, Computational evolution of human bipedal walking by a neuro-musculoskeletal model. Artif. Life Robot., 3(3), 133-138 (1999).

[16] G.W. Hiebert, P.J. Whelan, A. Prochazka, and K.G. Pearson, Contribution of hindlimb flexor muscle afferents to the timing of phase transitions in the cat step cycle. J. Neurophysiol., 75 , 1126-1137 (1996). 
[17] V.T. Inman, The pattern of muscular activity in the lower extremity during walking (Technical Report Series II, No. 25), Prosthetic Devices Research Project, Institute of Engineering Research, University of California, Berkeley, Calif (1953).

[18] M. Kawato, Transient and steady state phase response curves of limit cycle oscillators. J. Math. Biol., 12, 13-30 (1981).

[19] S. Jo and S.G. Massaquoi, A model of cerebrocerebello-spinomuscular interaction in the sagittal control of human walking. Biol. Cybern., 96, 279-307 (2007).

[20] M.A. Lewis, R. Etienne-Cummings, M.J. Hartmann, Z.R. Xu, and A.H. Cohen, An in silico central pattern generator: silicon oscillator, coupling, entrainment, and physical computation. Biol. Cybern., 88, 137-151 (2003).

[21] J. Nakanishi, J. Morimoto, G. Endo, G. Cheng, S. Schaal, and M. Kawato, Learning from demonstration and adaptation of biped locomotion. Robotics and Autonomous Systems, 47, 79-91 (2004).

[22] M. Nakanishi, T. Nomura, and S. Sato, Stumbling with optimal phase reset during gait can prevent a humanoid from falling. Biol. Cybern., 95, 503-515 (2006).

[23] N. Ogihara and N. Yamazaki, Generation of human bipedal locomotion by a bio-mimetic neuromusculo-skeletal model. Biol. Cybern., 84, 1-11 (2001).

[24] N. Ogihara, M. Nakatsukasa, Y. Sugimoto, S. Aoi, and K. Tsuchiya, Adaptive locomotion mechanisms inherent in the musculoskeletal structure, in Proc. SICE-ICASE Int. Conf., pp. 4495-4498 (2006).

[25] I.A. Rybak, K. Stecina, N.A. Shevtsova, and D.A. McCrea, Modelling spinal circuitry involved in locomotor pattern generation: insights from the effects of afferent stimulation. J. Physiol., $\mathbf{5 7 7}(2)$, 641-658 (2006)

[26] G. Taga, Y. Yamaguchi, and H. Shimizu, Self-organized control of bipedal locomotion by neural oscillators in unpredictable environment. Biol. Cybern., 65, 147-159 (1991).

[27] G. Taga, A model of the neuro-musculo-skeletal system for human locomotion I. Emergence of basic gait. Biol. Cybern., 73, 97-111 (1995).

[28] G. Taga, A model of the neuro-musculo-skeletal system for human locomotion II. - Real-time adaptability under various constraints. Biol. Cybern., 73, 113-121 (1995).

[29] T. Takuma and K. Hosoda, Controlling the walking period of a pneumatic muscle walker. Int. J. Robotics Res., 25(9), 861-866 (2006).

[30] N. Tomita and M. Yano, A model of bipedal walking controlled by the basal ganglia-brainstem systems, in Proc. 9th Int. Symp. Artif. Life Robot., pp. 359-362 (2004). 
[31] M. Vukobratović, B. Borovac, D. Surla, and D. Stokić, Biped locomotion dynamics, stability, control and application, Springer-Verlag (1990).

[32] P.J. Whelan, G.W. Hiebert, and K.G. Pearson, Stimulation of the group I extensor afferents prolongs the stance phase in walking cats. Exp. Brain Res., 103, 20-30 (1995).

[33] D.A. Winter, Biomechanics and motor control of human movement (3rd edition), New York: John Wiley \& Sons (2004).

[34] M. Wisse, G. Felksdal, J. van Frankenhuyzen and, B. Moyer, Passive-based walking robot. IEEE Robot. Autom. Mag., 14(2), 52-62 (2007).

[35] S. Yakovenko, V. Gritsenko, and A. Prochazka, Contribution of stretch reflexes to locomotor control: A modeling study. Biol. Cybern., 90, 146-155 (2004).

[36] T. Yamasaki, T. Nomura, and S. Sato, Phase reset and dynamic stability during human gait. BioSystems, 71, 221-232 (2003).

[37] T. Yamasaki, T. Nomura, and S. Sato, Possible functional roles of phase resetting during walking. Biol. Cybern., 88, 468-496 (2003). 